\title{
Circular RNA PRKCl promotes glioma cell progression by inhibiting microRNA-545
}

\author{
Xuebang Zhang ${ }^{1}$, Han Yang ${ }^{1}$, Lihao Zhao', Gang Li' and Yuxia Duan²
}

\begin{abstract}
We here tested expression and potential functions of circular RNA PRKCI (circPRKCl) in human glioma. Our results show that circPRKCl is upregulated in human glioma tissues and glioma cells, correlating with downregulation of its potential target, microRNA-545 (miR-545). In A172 and primary human glioma cells, shRNA-mediated silencing of circPRKCl inhibited cancer cell growth, survival, proliferation, and migration. Conversely, ectopic circPRKCl overexpression promoted A172 cell progression. miR-545 is the primary target of circPRKCl in glioma cells. Forced overexpression of miR-545 mimicked circPRKCl shRNA-induced actions, inhibiting glioma cell survival and proliferation. In contrast, miR-545 inhibition, by a lentiviral antagomiR-545 construct, reversed circPRKCl shRNA-induced anti-A172 cell activity. Importantly, neither circPRKCI shRNA nor circPRKCl overexpression was effective in miR-545-knockout (Cas9 method) A172 cells. Importantly, the subcutaneous and orthotopic A172 xenograft growth was significantly inhibited by circPRKCI silencing. Collectively, circPRKCl promotes human glioma cell progression possibly by inhibiting miR-545. Targeting circPRKCl-miR-545 cascade could efficiently inhibit human glioma cells.
\end{abstract}

\section{Introduction}

Glioma, the most common brain tumor and a global health threat, causes significant mortalities each year ${ }^{1-3}$. Current therapeutic options for glioma have failed to substantially improve patients' prognosis ${ }^{4-6}$. High-grade (grade III-IV) glioma (i.e., glioblastoma) has one of the worst survival among all malignancies ${ }^{1-3}$, which is possibly due to its molecular heterogeneity ${ }^{1}$. It is therefore urgent to further explore the pathological mechanisms for glioma progression, which could help to develop novel and more efficient molecularly-targeted therapies $^{7-9}$.

microRNA (miRNAs), long non-coding RNAs (LncRNAs), and circular RNAs (circRNAs) are noncoding $\mathrm{RNAs}^{10,11}$. Recent studies have proposed the pivotal roles of these non-coding RNAs in glioma

\footnotetext{
Correspondence: Gang Li (ligangwzyd@163.com) or

Yuxia Duan (wydyx59@163.com)

'Department of Chemoradiation Oncology, The first affiliated hospital of

Wenzhou Medical University, Wenzhou, Zhejiang, P.R. China

${ }^{2}$ Department of Radiology, The First Affiliated Hospital of Wenzhou Medical

University, Wenzhou, Zhejiang, P.R. China

These authors contributed equally: Xuebang Zhang, Han Yang

Edited by A. Stephanou
}

progression $^{12}$. Unlike other non-coding RNAs, circRNAs can form highly stable circular structure via joining of the $3^{\prime}$ and $5^{\prime}$ terminals ${ }^{11,13,14}$. circRNAs function as miRNAs sponges, regulating gene expression in cancer cells ${ }^{11,14,15}$. Dysregulation of circRNAs is often detected in glioma ${ }^{12}$.

Qiu et al. have recently discovered a novel and oncogenic circRNA, namely circRNAs PRKCI (circPRKCI) ${ }^{16}$. circPRKCI, with 1,484-bp long, is back-spliced of two exons (15 and 16) of PRKCI gene located at 3q26.2 ${ }^{16}$. circPRKCI is upregulated in lung adenocarcinoma in part due to the amplification of 3q26.2 locus, promoting cancer cell proliferation and tumorigenesis ${ }^{16}$. circPRKCI is mainly present in the cytoplasm, sponging miR-545 and miR-589, thereby abolishing the suppressing of their target, the transcription factor $E 2 F 7^{16}$. Shi et al. have shown that circPRKCI promotes AKT3 expression and esophageal squamous cell proliferation by sponging miR-3680$3 \mathrm{p}^{17}$. In human hepatocellular carcinoma (HCC) cells circ-PRKCI functions as the sponge of miR-545, promoting cell survival ${ }^{18}$.The expression and potential functions of circPRKCI in human glioma cells are tested in the present study.

\section{(c) The Author(s) 2019}

(c) Open Access This article is licensed under a Creative Commons Attribution 4.0 International License, which permits use, sharing, adaptation, distribution and reproduction cc) in any medium or format, as long as you give appropriate credit to the original author(s) and the source, provide a link to the Creative Commons license, and indicate if changes were made. The images or other third party material in this article are included in the article's Creative Commons license, unless indicated otherwise in a credit line to the material. If material is not included in the article's Creative Commons license and your intended use is not permitted by statutory regulation or exceeds the permitted use, you will need to obtain permission directly from the copyright holder. To view a copy of this license, visit http://creativecommons.org/licenses/by/4.0/. 


\section{Materials and methods}

\section{Chemicals and reagents}

The antibodies utilized in this study were purchased from Abcam (Cambridge, MA). The reagents for cell culture were purchased from Hyclone (Logan, UT). Puromycin, polybrene and all other chemicals were provided by Sigma-Aldrich (St. Louis, Mo). All primers, sequences, virus, and expression constructs were designed, provided, and sequence verified by Shanghai Genechem Co. (Shanghai, China).

\section{Cell culture}

The established A172 glioma cells were provided by Dr. $\mathrm{Cao}^{19}$. Brain cortical tissues were obtained from 15-weekold normal fetal brains (prepared from Dr. Zhang ${ }^{20}$ ), dissociated, digested, and filtered as previously described $^{21,22}$. The dissociated cells were centrifuged and resuspended in MEM medium with applied supplements ${ }^{22}$. Cells were seeded at a density of $2 \times 10^{6}$ cells $/ \mathrm{mL}$ on poly-lysine-coated tissue culture flasks. For neuronal culture, astrocytes were limited by 4-day treatment with FDU (Sigma). Neuronal cultures were grown for eight days (day in vitro 8, DIV8) before any further experiments. The primary human astrocytes $(95 \%$ positive for glial fibrillary acidic protein) were provided from Dr. $\mathrm{Cao}^{19}$, prepared from 12-week cortical tissues from normal fetal brains. All procedures were approved by the Ethics Committee of Wenzhou Medical University.

\section{Primary human glioma cells and tissues}

In this study the primary human glioma cells were provided by Dr. $\mathrm{CaO}^{19,23}$, derived from three writteninformed consent glioma patients, named as "Pri-1/-2/-3". Primary human glioma cells were cultured in complete RPMI medium with necessary supplements and antibiotics $^{24,25}$. The human glioma tissues and paired paracancer normal brain tissues were again provided by Dr. $\mathrm{Cao}^{19,23}$, stored in liquid nitrogen and subjected to further biomedical analyses. The protocols of this study were approved by the Ethics Committee of Wenzhou Medical University, in according to Declaration of Helsinki.

\section{Quantitative real-time PCR (qPCR)}

Total cellular and tissue RNAs were isolated by the Trizol reagent (Invitrogen, Grand Island, NY) and quantified. mRNA expression was tested by using the SYBR GREEN PCR Master Mix (Applied Biosystems, Beijing, China) under an ABI 7600 fast Real-time PCR System (Applied Biosystems). Relative expression of targeted mRNAs was calculated by $2^{-\Delta \Delta C t}$ method, using GAPDH as the internal control. circPRKCI and miR-545 levels were tested by the TransStartTM SYBR Green qPCR Supermix (TransGen Biotech, Beijing, China), using U6 small nuclear RNA as the internal control. All the primers were listed in Table. 1.

\section{Western blotting}

Equivalent amounts of total cellular lysates $(40 \mu \mathrm{g}$ per treatment) were separated by $10-12 \%$ of SDS-PAGE gels, then transferred to the polyvinylidene fluoride (PVDF) blots (Merck Millipore, Darmstadt, Germany). After blocking in $10 \%$ non-fat milk, the blots were incubated with the applied primary antibodies, followed by incubation with corresponding secondary antibodies. Antibodyantigen binding was detected by an enhanced chemiluminescence (ECL) substrate kit (Invitrogen), with the results quantified by an ImageJ software $(\mathrm{NIH}$, Bethesda, MD).

\section{Cell Counting Kit-8 (CCK-8) assay}

Cells were initially seeded into 96 -well plates at $5 \times 10^{3}$ cells per well. Following incubation for $72 \mathrm{~h}$, CCK-8 solution $(10 \mu \mathrm{L} /$ well, Dojindo Molecular Technologies, Gaithersburg, MD) was added to each well. After incubation for another $3 \mathrm{~h}$, CCK- 8 optical density (OD) values were measured at test wavelength of $450 \mathrm{~nm}$.

\section{Colony formation assay}

A172 cells were initially seeded at $1 \times 10^{4}$ cells per 10 $\mathrm{cm}$ dish. Colony formation assays were conducted for 10 days, and the colonies were fixed and stained (with 1\% crystal violet solution). The number of colonies was counted manually.

Table. 1 Primer sequences of the study

\begin{tabular}{|c|c|c|}
\hline Genes & Forward primer & Reverse primer \\
\hline circPRKCl & 5'-ATTCAGGGACACCCGTTCTT-3' & 5'-CTCTTCAGAACACTTGCAGCTT-3' \\
\hline U6 & 5'-CTCGCTTCGGCAGCACA-3' & 5'-AACGCTTCACGAATTTGCGT-3' \\
\hline GAPDH & 5'-CGCTCTCTGCTCCTCCTGTTC-3' & 5'-ATCCGTTGACTCCGACCTTCAC-3' \\
\hline E2F7 & 5'-CTGCTGCGCTAGACTTGGAT-3' & 5'-TCTCTTAGTAGGACCACCAACG-3' \\
\hline$R / G-1$ & 5'-AGCACTGGGTGCATGAGGCCT-3' & 5'- TCCTITGTGGCAGCACCCAATG-3' \\
\hline $\operatorname{miR}-545$ & 5'-ACGGCCATACCACCCTGAAC-3' & 5'-GGCGGTCTCCCATCCAAGTA-3' \\
\hline
\end{tabular}




\section{In vitro migration assay}

A172 cells $\left(1 \times 10^{5}\right.$ cells in $300 \mu \mathrm{L}$ serum-free medium) were seeded into the upper part of each "Transwell" chambers (12- $\mu \mathrm{m}$ pore size, BD Biosciences, Heidelberg, Germany). The lower compartments were filled with medium with $10 \%$ FBS. Following incubation for $24 \mathrm{~h}$, nonmigrated cells on the upper surface were wiped out. The migrated cells, on the lower surface, were fixed and stained.

\section{EdU assay of proliferation}

Cells were seeded into six-well plates at $6 \times 10^{4}$ cells per well, and cultured for $48 \mathrm{~h}$. An EdU (5-ethynyl-20-deoxyuridine) Apollo-567 Kit (RiboBio, Guangzhou, China) was applied. EdU and DAPI dyes were added to glioma cells for additional $4 \mathrm{~h}$. Under a fluorescent microscope cell nuclei were visualized. For each condition total 300 nuclei in five random views were included to calculate EdU ratios $($ EdU/DAPI $\times 100 \%)$.

\section{Annexin V FACS assay}

Following the applied genetic treatments, Annexin VFITC and Propidium Iodide (PI) dyes (each at $10 \mu \mathrm{g} / \mathrm{mL}$, BD Pharmingen, San Diego, CA) were added for $30 \mathrm{~min}$ under the dark at room temperature. Cell apoptosis was analyzed by a flow cytometry machine (Beckman Coulter, Brea, CA).

\section{circPRKCI ShRNA}

Two shRNAs targeting non-overlapping sequences ("Seq-1/2") of circPRKCI were individually sub-cloned into GV248 (hU6-MCS-Ubiquitin-EGFP-IRES-puromycin) construct (Shanghai Genechem Co.), then transfected to HEK-293 cells with lentivirus package plasmid mix (Shanghai Genechem Co.). The generated circPRKCI shRNA lentivirus ("LV-circPRKCI shRNA") was added to cultured glioma cells (in polybrene medium). Following selection by puromycin $(5.0 \mu \mathrm{g} / \mathrm{mL}$, for 4-5 passages), stable cells were established. Silencing of circPRKCI (over 90\% knockdown efficiency) in stable cells was confirmed by qPCR. Control cells were transfected with lentiviral scramble control shRNA.

\section{Ectopic overexpression of circPRKCl or miR-545}

The full-length circPRKCI ${ }^{16,26}$ and pri-miR-545 $5^{16,26}$ were synthesized by Shanghai Genechem Co, sub-cloned to a lentiviral GV248 construct (Shanghai Genechem Co.). The construct, together with lentivirus package plasmid mix, was co-transfected to HEK-293 cells to generate circPRKCI-expressing lentivirus ("LV-circPRKCI") and miR-545-expressing lentivirus ("LV-miR-545"). Following filtration and enrichment, LV-circPRKCI or LV-miR-545 was added to cultured glioma cells. Puromycin was added to select stable cells. Control cells were infected with lentivirus with empty vector.

\section{miR-545 inhibition}

The miR-545 inhibitor precursor, provided by Shanghai Genechem, was sub-cloned into the GV248 vector, cotransfected into $293 \mathrm{~T}$ cells with lentivirus package plasmids to generate antagomiR-545 lentivirus (LV-antagomiR-545). The latter was transduced to A172 cells for $24 \mathrm{~h}$. Puromycin was added again to select the stable cells. miR-545 inhibition in the stable cells was confirmed by qPCR.

\section{miR-545 knockout}

The CRISPR/Cas9 miR-545-KO lentivirus, with sgRNA targeting the pri-miR-545 sequence, was synthesized and verified by Shanghai Genechem Co. (Shanghai, China), added to A172 cells. The infected cells were than cultured in puromycin selection medium until stable cells were achieved. Cells were further transfected with or without the lentiviral circPRKCI shRNA construct ("LV-circPRKCI shRNA") or the lentiviral circPRKCI overexpression construct ("LV-circPRKCI"). Expression of miR-545 and circPRKCI was tested by qPCR.

\section{RNA immunoprecipitation (RIP)}

A172 cells were lysed in complete RIP lysis buffer (purchased from Beyotime Biotechnology, Suzhou, China), and the cell extracts ( $800 \mu \mathrm{g}$ lysates per treatment) were incubated with the magnetic beads conjugated with anti-Argonaute 2 (Ago2, Sigma) or control anti-IgG antibody (Sigma) for $12 \mathrm{~h}$. The beads were washed and incubated with Proteinase K. Finally, the purified RNA was subjected to qPCR analysis, with results normalized to the Input control.

\section{A172 xenograft assay}

The severe combined immunodeficient (SCID) mice (4-5 week old, about $18 \mathrm{~g}$ weight) were provided by the Animal Center of Suzhou University (Suzhou, China), inoculated via subcutaneous (s.c.) injection with five million A172 cells with circPRKCI shRNA or control shRNA, in $200 \mu \mathrm{L}$ of Matrigel gel/basal medium. Mouse body weights and tumor volumes were measured weekly, and tumor volume calculated by a described formula ${ }^{19}$. For intracranial tumor implantation, A172 glioma cells $\left(1 \times 10^{6}\right.$ cells $)$ were implanted as described ${ }^{27}$. All animal procedures were approved by the Ethics Committee of Wenzhou Medical University.

\section{Statistics}

Statistical analyses were performed by SPSS 21.0 software (SPSS Inc., Chicago, IL). All data were presented as mean \pm standard deviation (SD). Statistical differences were performed by one-way ANOVA within multiple comparisons with post hoc Bonferroni test. To determine significance between two treatment groups, the two-tailed 
$t$-tests were carried out. $P$ values $<0.05$ were considered statistically significant.

\section{Results}

circPRKCl is upregulated in human glioma tissues and cells

First, circPRKCI expression in human glioma tissues was examined. A total of five pairs of fresh glioma tissues (“T”, from Dr. $\mathrm{Cao}^{19}$ ) and parecancer normal brain tissues ("N") were obtained. qPCR assays were performed to test circPRKCI expression. Results, in Fig. 1a, demonstrated that circPRKCI levels are significantly upregulated in all tested glioma tissues, when compared its levels in the normal brain tissues. Furthermore, circPRKCI is upregulated in A172 glioma cells and in the primary human glioma cells ("Pri-1/-2/-3", see Methods) (Fig. 1b). While its levels are low in primary human neuronal cultures and human astrocytes (Dr. Cao ${ }^{19}$ ) (Fig. 1b).

It has been previously shown that circPRKCI functions as the sponge of miR-545, a tumor-suppressive miRNA ${ }^{16}$. We therefore tested miR-545 expression in glioma tissues and cells. As demonstrated, miR-545 levels are significantly downregulated in glioma tissues (Fig. 1c), as well as in the established and primary human glioma cells (Fig. 1d). In contrast, miR-545 expression is relatively high in normal brain tissues, primary human neuronal cultures and human astrocytes (Fig. 1c, d). Another important miRNA target of circPRKCI, miR-589 ${ }^{16}$, was not detected in our system. These results show that circPRKCI is
A.

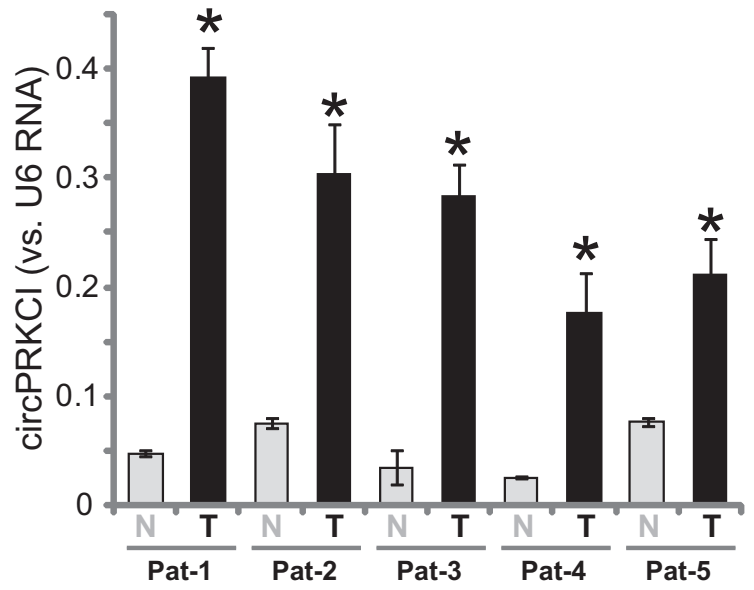

C.

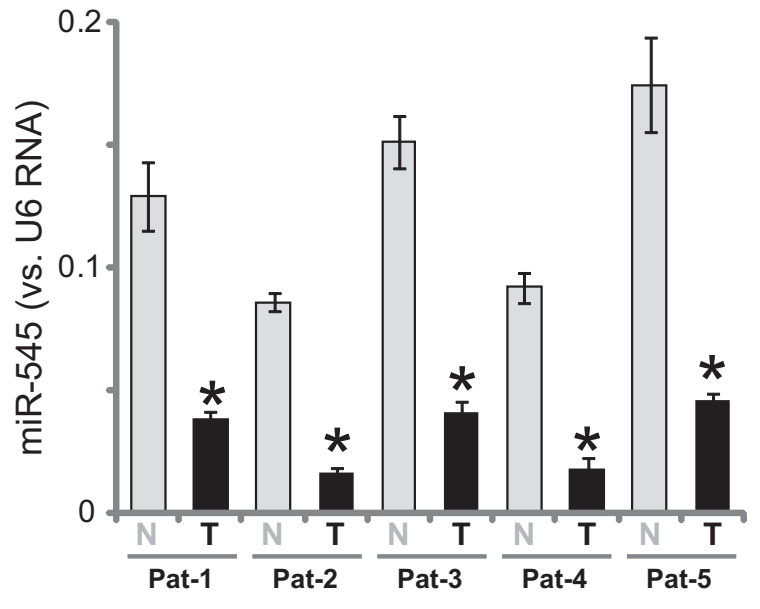

B.

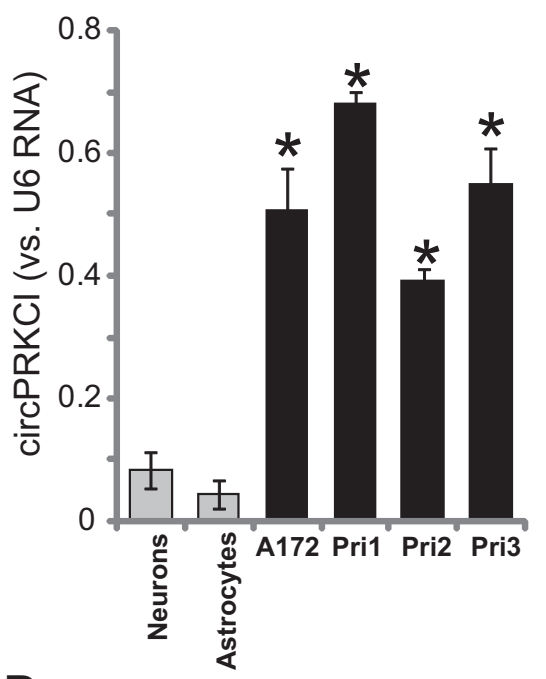

D.

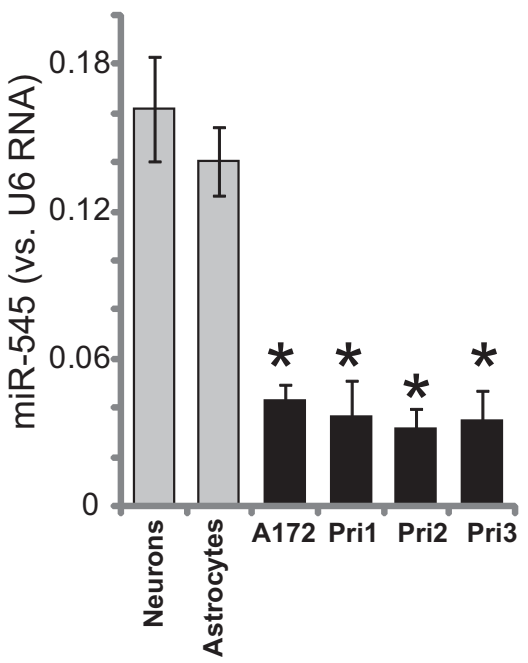

Fig. 1 circPRKCl is upregulated in human glioma tissues and cells. Total RNA was extracted from the described human tissues and cells, expression of circPRKCl $(\mathbf{a}, \mathbf{b})$ and miR-545 ( $\mathbf{c}, \mathbf{d})$ was tested by qPCR assays, results were normalized to U6 RNA. "Pat" stands for glioma patient number. Error bars stand for mean \pm standard deviation $(S D, n=5)$. ${ }^{*} P<0.05$ vs. "N" tissues $(\mathbf{a}, \mathbf{c})$ or primary neuronal culture ("Neurons") $(\mathbf{b}, \mathbf{d})$ 
upregulated in human glioma tissues and cells, correlating with miR-545 downregulation.

circPRKCl shRNA inhibits A172 glioma cell growth, survival, proliferation, and migration

In order to study the potential function of circPRKCI in glioma cells, a shRNA strategy was applied. GV248 lentiviral shRNA, targeting non-overlapping sequence ("Seq1/Seq-2") against circPRKCI ("sh-circPRKCI"), was added to A172 glioma cells. Following puromycin selection stable cells were established. Testing circPRKCI expression in the stable cells, by qPCR assays, confirmed that circPRKCI levels decreased over 90\% (vs. the parental control cells) (Fig. 2a). By counting cell number, we show that circPRKCI shRNA-expressing A172 cells grew significantly slower than the control cells (Fig. 2b). Cell viability, or the CCK-8 OD, was decreased as well by circPRKCI shRNA (Fig. 2c). Further experimental results demonstrated that circPRKCI shRNA decreased the number of A172 cell colonies (Fig. 2d) and EdU incorporation (Fig. 2e), indicating its anti-proliferative activity. Expression of proliferation marker proteins, including cyclin D1 and proliferating cell nuclear antigen (PCNA), was significantly downregulated as well in circPRKCIsilenced A172 glioma cells (Fig. 2f).

"Transwell" assay was performed to test cell migration in vitro. Results demonstrated that following circPRKCI silencing, the number of migrated A172 cells was significantly reduced (Fig. 2g). Additional studies showed that circPRKCI shRNA induced apoptosis activation in A172 cells, evidenced by increased Annexin V staining (Fig. 2h) as well as cleavages of caspase-3, caspase-9 and PARP [poly (ADP-ribose) polymerase] (Fig. 2i). The nonsense scramble control shRNA lentivirus ("sh-C"), as expected, exerted no significant effect on circPRKCI expression (Fig. 2a) nor A172 cell functions (Fig. 2b-i). Collectively, these results show that circPRKCI silencing inhibited cell growth, survival, proliferation, and migration, whiling inducing apoptosis activation in A172 glioma cells.

\section{circPRKCI silencing inhibits primary human glioma cell progression}

Next, we studied the potential function of circPRKCI in primary cells. The primary human glioma cells, derived three different patients ("Pri-1/-2/-3", see Fig. 1), were transduced with lentiviral circPRKCI shRNA ("Seq-1", see Fig. 2). Following selection by puromycin stable cells were established, showing $80-90 \%$ reduction of circPRKCI expression (Fig. 3a). shRNA-induced knockdown of circPRKCI in the primary glioma cells induced significant viability reduction (Fig. 3b), proliferation inhibition (Fig. 3c), cell migration inhibition (Fig. 3d) and apoptosis activation (Fig. 3e). Importantly, circPRKCI shRNA failed to affect the viability (Fig. 3f) and apoptosis (Fig. 3g) in primary human neuronal cultures ("Neurons") and primary human astrocytes ("Astrocytes"), where circPRKCI levels are low (Fig. 1), indicating a cancer cell specific effect by circPRKCI.

Next, a lentiviral circPRKCI expression construct ("LVcircPRKCI") was transduce to A172 cells. Two lines of stable cells, "Line-1" and "Line-2", were established with selection by puromycin, showing $8-10$ folds increase of circPRKCI expression (Fig. 3h). LV-circPRKCI-expressing cells presented with increased cell viability (CCK-8 OD, Fig. 3i) and proliferation (EdU ratio, Fig. 3j), as compared to the control A172 cells with empty vector. Therefore, forced overexpression of circPRKCI promoted A172 cell progression, further confirming its oncogenic role in glioma cells.

miR-545 is the primary target of circPRKCl in glioma cells

It has been shown that circPRKCI sponges miR- $545^{16}$, a tumor-suppressive microRNA ${ }^{16,28,29}$. We first explored the potential function of miR-545 in glioma cells. A lentiviral pri-miR-545 construct (LV-miR-545) was transfected to A172 cells. Following selection by puromycin two stable cell lines ("Line-1/-2") were established, showing significantly increased miR-545 expression (Fig. 4a). Expression of miR-545 targets, including the transcription factor E2F7 and RIG-1 (retinoic acidinducible gene-I) $)^{16,28}$, was largely downregulated (Fig. 4b, c). Forced expression of miR-545 inhibited A172 cell proliferation (EdU ratio, Fig. 4d), mimicking actions by circPRKCI shRNA (Fig. 2).

The Argonaute 2 (Ago2) protein can bind to both circRNAs and miRNAs, initiating the ceRNA mechanism and miRNA sponging ${ }^{16,30-32}$. In A172 glioma cells the RIP assay results revealed that circPRKCI and miR-545 were both efficiently pulled down by the anti-Ago 2 antibody, but not by the non-specific anti-IgG antibody (Fig. 4e). This is consistent with previous findings in lung adenocarcinoma cells ${ }^{16}$. Indeed, in the circPRKCI shRNAexpressing A172 cells, miR-545 levels were significantly increased (Fig. 4f), causing E2F7 and RIG-1 downregulation (Fig. 4g). Significantly, inhibition of miR-545, by a lentiviral miR-545 inhibitor construct ("LV-antagomiR-545") (Fig. 4f), completely reversed E2F7 and RIG-1 inhibition by circPRKCI shRNA (Fig. 4g). Significantly, in A172 cells circPRKCI shRNA-induced viability reduction (Fig. 4h) and proliferation inhibition (Fig. 4i) were nullified by LV-antagomiR-545. LV-antagomiR-545 by itself enhanced E2F7/RIG-1 expression (Fig. 4g), A172 cell viability (Fig. 4h) and proliferation (Fig. 4i). These results indicate that circPRKCI possibly sponges tumorsuppressive miR-545 in A172 cells. Conversely, circPRKCI shRNA inhibited A172 cell progression by restoring miR-545 expression. 


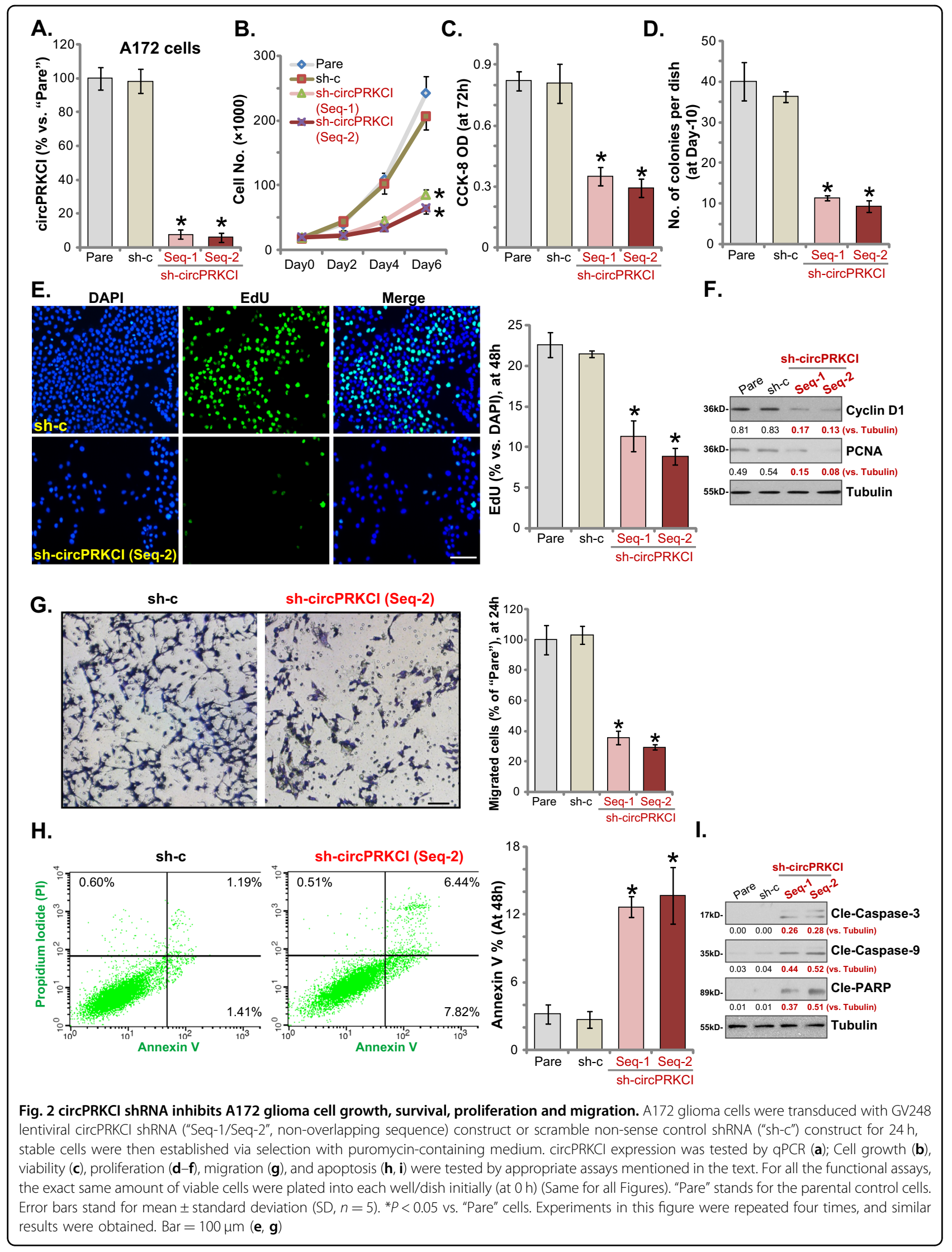


A.

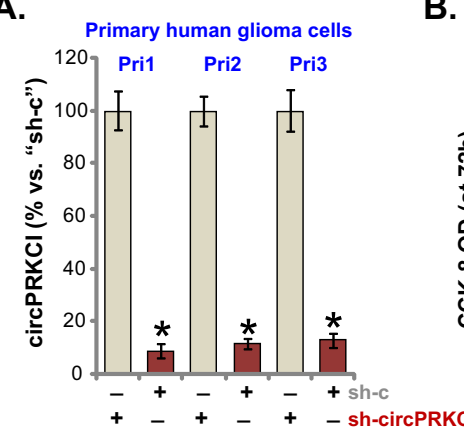

D.

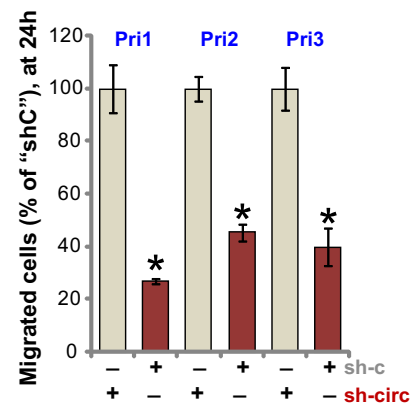

B.

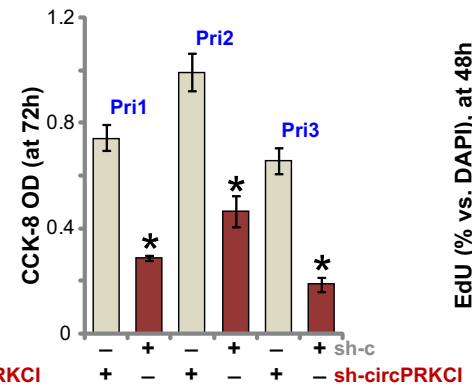

C.
E.

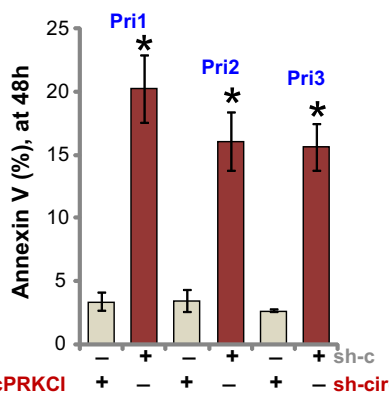

F.

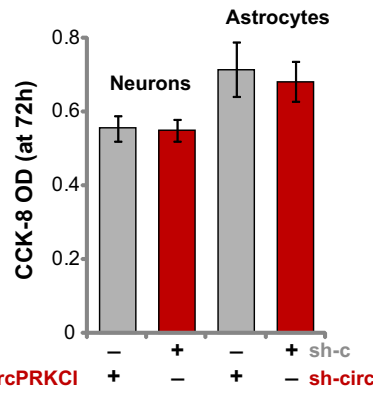

G.

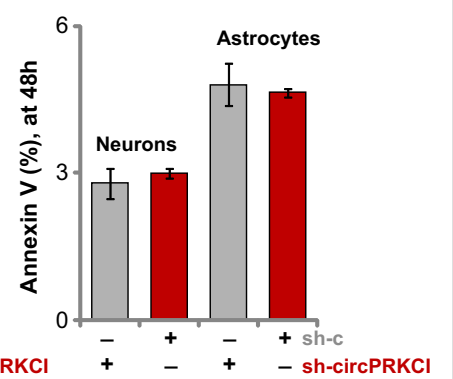

H.

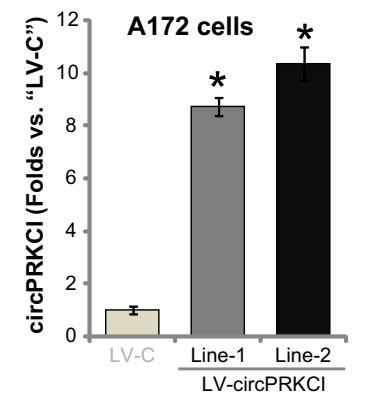

I.

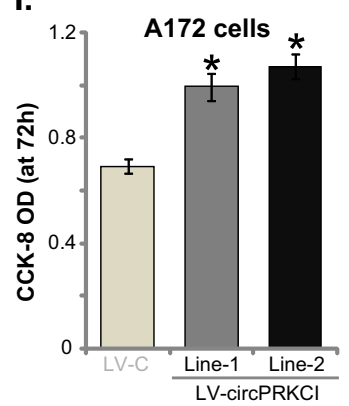

J.

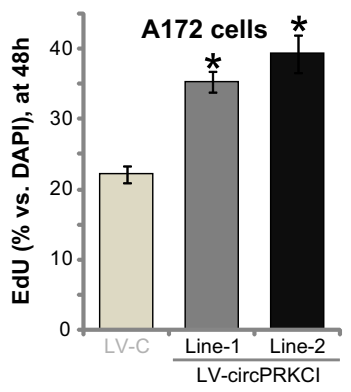

Fig. 3 circPRKCl silencing inhibits primary human glioma cell progression. The primary human glioma cells, derived from three different patients ("Pri-1/-2/-3"), as well as primary human neuronal cultures ("Neurons") and primary human astrocytes ("Astrocytes"), were transduced with GV248 lentiviral circPRKCI shRNA ("Seq-1") or scramble non-sense control shRNA ("sh-c") for $24 \mathrm{~h}$, stable cells were established via selection with puromycin. circPRKCl expression was tested by qPCR (a); Cell viability (CCK-8 OD, b, f), proliferation (EdU ratio, $\mathbf{c}$ ), migration ("Transwell" assay, $\mathbf{d}$ ), and apoptosis (Annexin $V$ ratio, e, g) were tested. A172 cells were transduced with lentiviral circPRKCl expression construct ("LV-circPRKCI") or empty vector ("LV-C") for 24 h, stable cells were established via puromycin selection; circPRKCl expression (h), cell viability (i), and proliferation (j) were tested similarly. Error bars stand for mean \pm standard deviation $(S D, n=5)$. ${ }^{*} P<0.05$ vs. "sh-C"/"LV-C" cells. Experiments in this figure were repeated four times, and similar results were obtained

To further confirm that miR-545 is the primary target of circPRKCI, the CRISPR/Cas9 method was applied to completely and stably knockout pri-miR-545 in A172 cells (Fig. 4j). As compared to the control cells, miR-545-KO A172 cells showed increased cell viability (Fig. 4k) and proliferation (Fig. 4l). Importantly, neither LV-circPRKCI nor circPRKCI shRNA was effective in the miR-545-KO cells (Fig. 4k, l), although both did significantly change circPRKCI expression (Fig. 4m). These results confirm that miR-545 is the primary target of circPRKCI in mediating its actions in glioma cells.

\section{circPRKCl silencing inhibits subcutaneous A172 glioma growth in SCID mice}

To study the potential function of circPRKCI in vivo, stable A172 glioma cells, with circPRKCI shRNA ("Seq-1/ Seq-2") or scramble non-sense control shRNA ("sh-c"), were s.c. injected to the flanks of SCID mice. Within 3 weeks the subcutaneous A172 tumors were established, with tumor volumes close to $100 \mathrm{~mm}^{3}$ (labeled as Day-0/ "D0"). Recording weekly tumor volumes, in Fig. 5a, demonstrated that A172 xenografts with circPRKCI shRNA grew significantly slower than the control tumors 


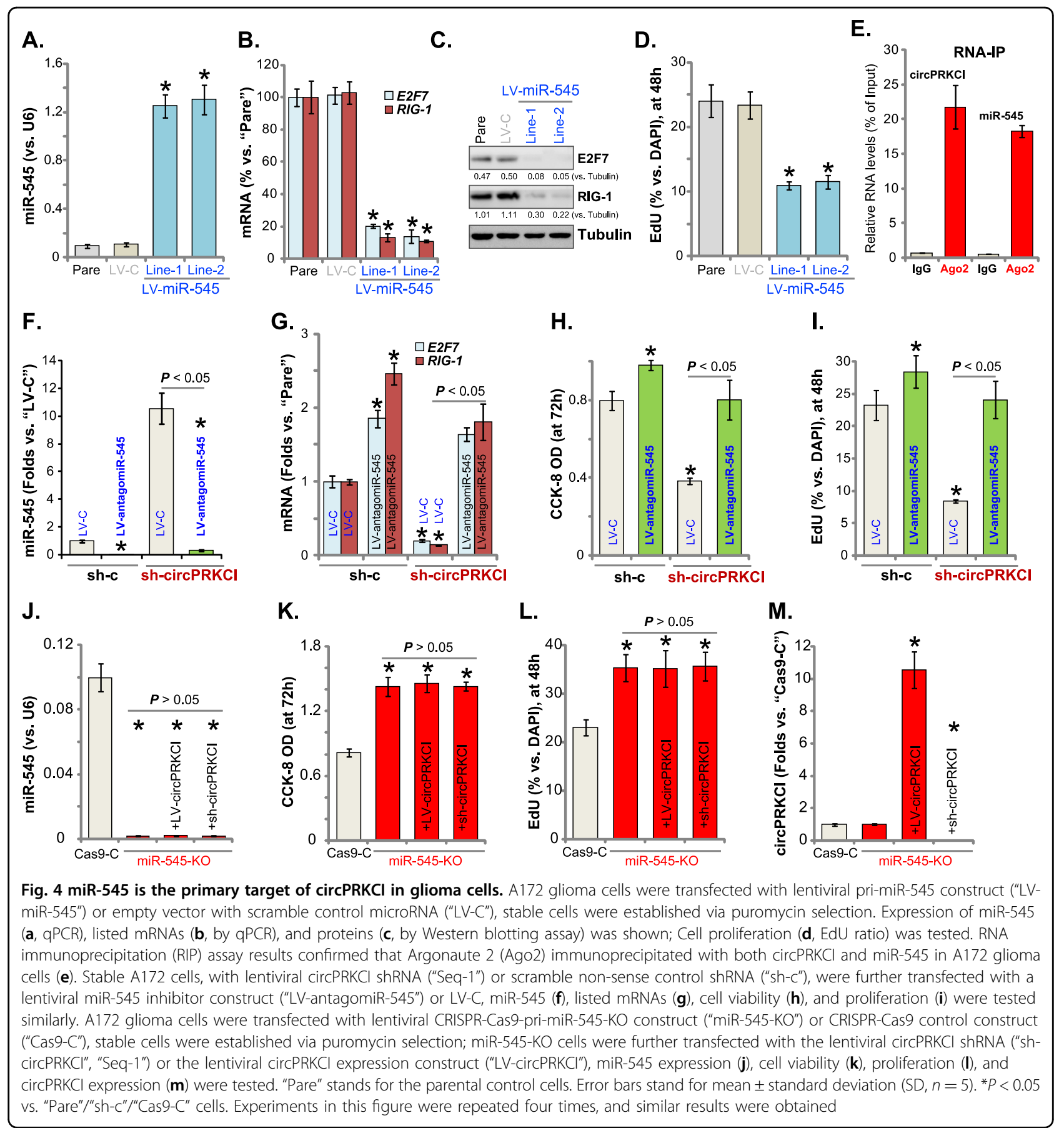

(with "sh-c"). When calculating the estimated daily tumor growth, using the formula [tumor volume at Day-35 (D35) subtracting tumor volume at Day- $0(\mathrm{D} 0)] \div 35$, we showed again that subcutaneous A172 tumor growth was significantly inhibited by circPRKCI shRNA (Fig. 5b). At D35, tumors of all three groups were isolated and weighted. Results confirmed that circPRKCI shRNAexpressing A172 tumors weighted significantly lower than the control tumors (Fig. 5c). The mice body weights were not significantly different between the three groups (Fig. 5d), neither did we notice any signs of apparent toxicities in the experimental mice.

At D7 and D14, one tumor of each group was isolated, and tumor tissues were achieved and analyzed. Testing circPRKCI expression, by qPCR, demonstrated that circPRKCI levels were significantly downregulated in tumors with circPRKCI shRNA (Fig. 5e). On the contrary, miR545 levels were dramatically increased (Fig. 5f). Protein 


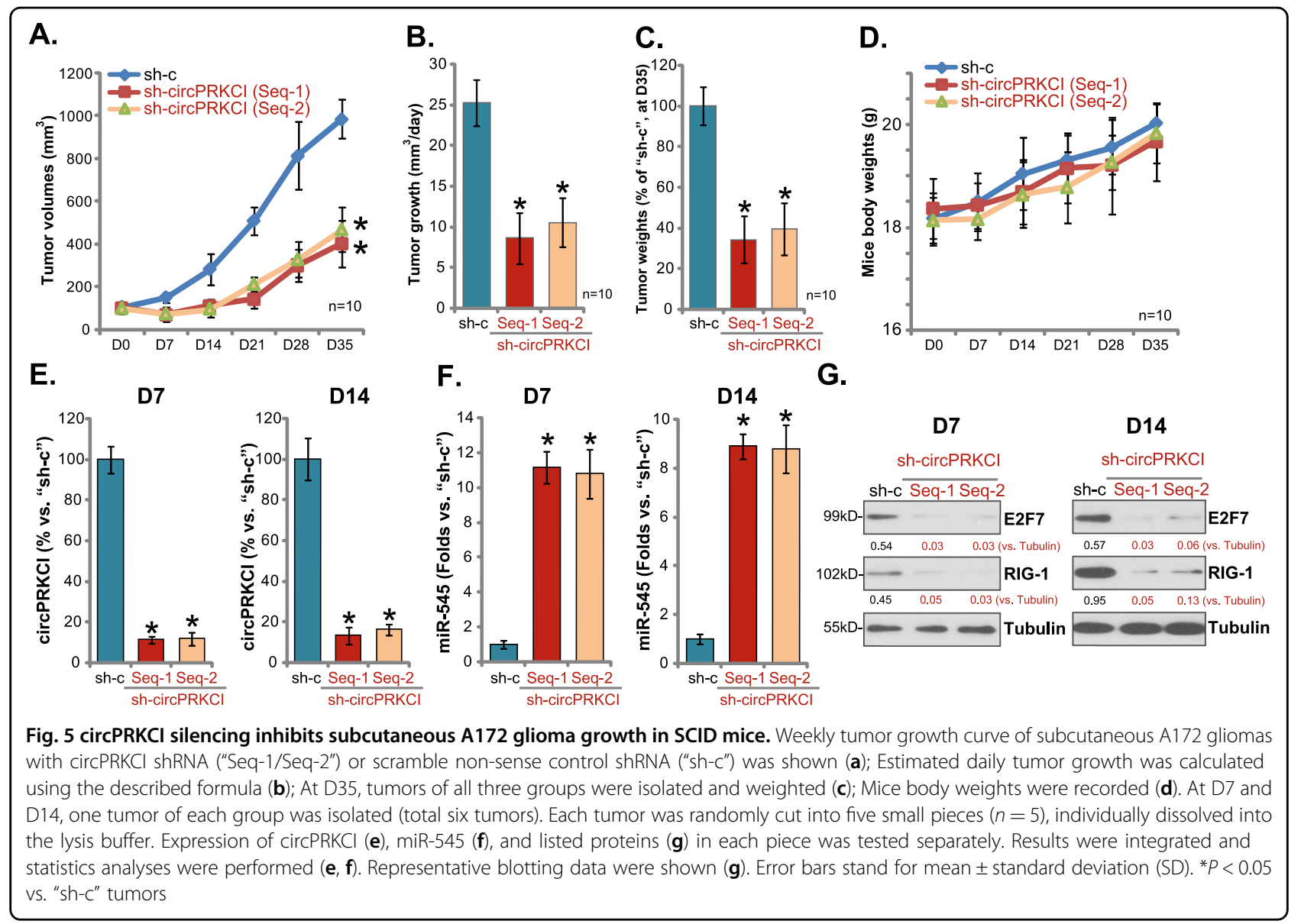

expression of miR-545 targets, E2F7 and RIG-1, was downregulated in circPRKCI-silenced tumors (Fig. 5g). Thus, in line with the in vitro findings, circPRKCI shRNA induced miR-545 accumulation, E2F7 and RIG-1 downregulation in subcutaneous A172 glioma tumors.

\section{circPRKCl shRNA inhibits orthotopic A172 glioma growth in mice}

To further study a role of circPRKCI in glioma cell progression, orthotopic glioma xenografts were established. Exact same number of A172 cells $\left(1 \times 10^{6}\right.$ cells of each mouse mouse) with circPRKCI shRNA ("Seq-1/Seq2") or scramble non-sense control shRNA ("sh-c"), were intracranially injected to the brain of SCID mice ${ }^{19}$. At day-21, with first mouse in the control tumor group showed the typical neurologic and sick symptoms, all groups were sacrificed, with tumors isolated. Results of tumor volumes (Fig. 6a) and the tumor weights (Fig. 6b) demonstrated that orthotopic A172 gliomas with circPRKCI shRNA grew significantly slower than the control tumors (Fig. 6a, b). Once again, the mice body weights were not significantly different between the three groups (Fig. 6c). Biochemical analyses of tumor tissue lysates confirmed that circPRKCI levels were significantly downregulated in the orthotopic A172 xenografts with circPRKCI shRNA (Fig. 6d), whereas miR545 levels significantly increased (Fig. 6e). miR-545 targets, E2F7 and RIG-1, were downregulated in circPRKCI-silenced orthotopic tumor tissues (Fig. 6f). Therefore, circPRKCI shRNA inhibited orthotopic A172 glioma growth in mice.

\section{Discussion}

circRNAs are a large and conserved family of noncoding RNAs, generated from a non-canonical back splicing process, from a covalent bond between $5^{\prime}$ and $3^{\prime}$ ends of a single-stranded RNA ${ }^{14,33}$. Dysregulation of circRNAs has been detected in glioma cells, essential for cancer development and progression ${ }^{12}$. Our results suggest that circPRKCI is an oncogenic circRNA in glioma. Its expression is upregulated in human glioma tissues and in established/primary human glioma cells, but low in normal brain tissues and neurons/astrocytes. In A172 cells and primary human glioma cells, circPRKCI silencing, by targeted shRNA, potently inhibited cell growth, survival, proliferation, and migration, whiling inducing apoptosis activation. Conversely, exogenous overexpression of circPRKCI, by a lentiviral construct, promoted A172 cell progression. Furthermore, in vivo growth 

A.
B.
C.

\section{Orthotopic A172 xenografts}
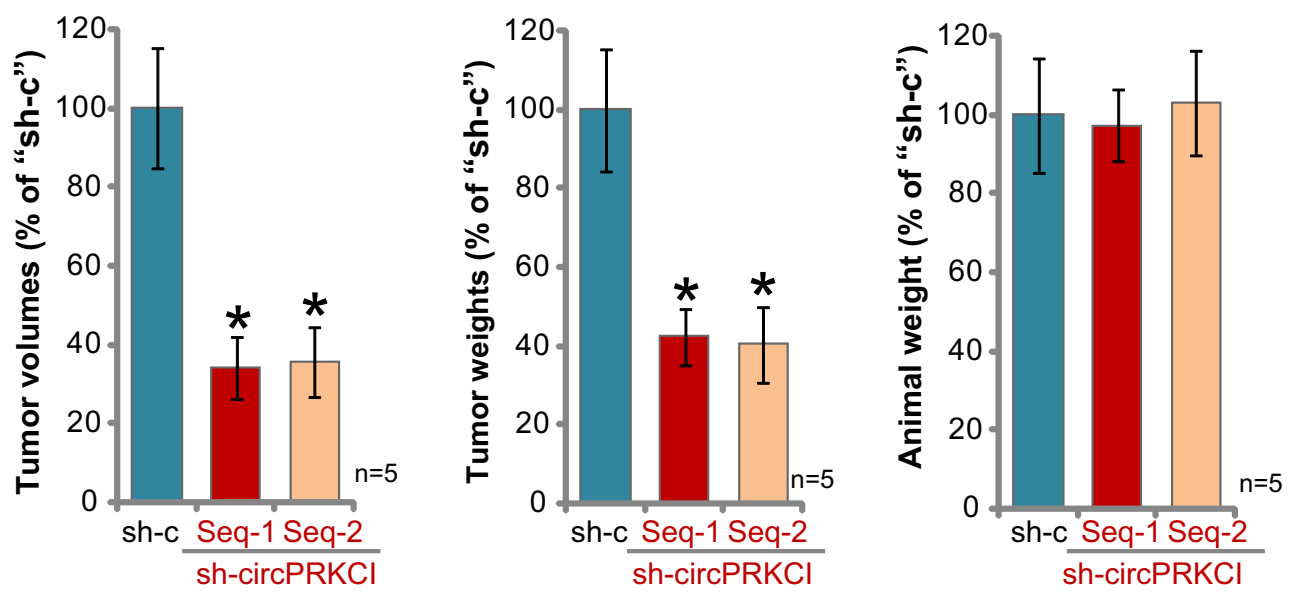

D.

E.

F.
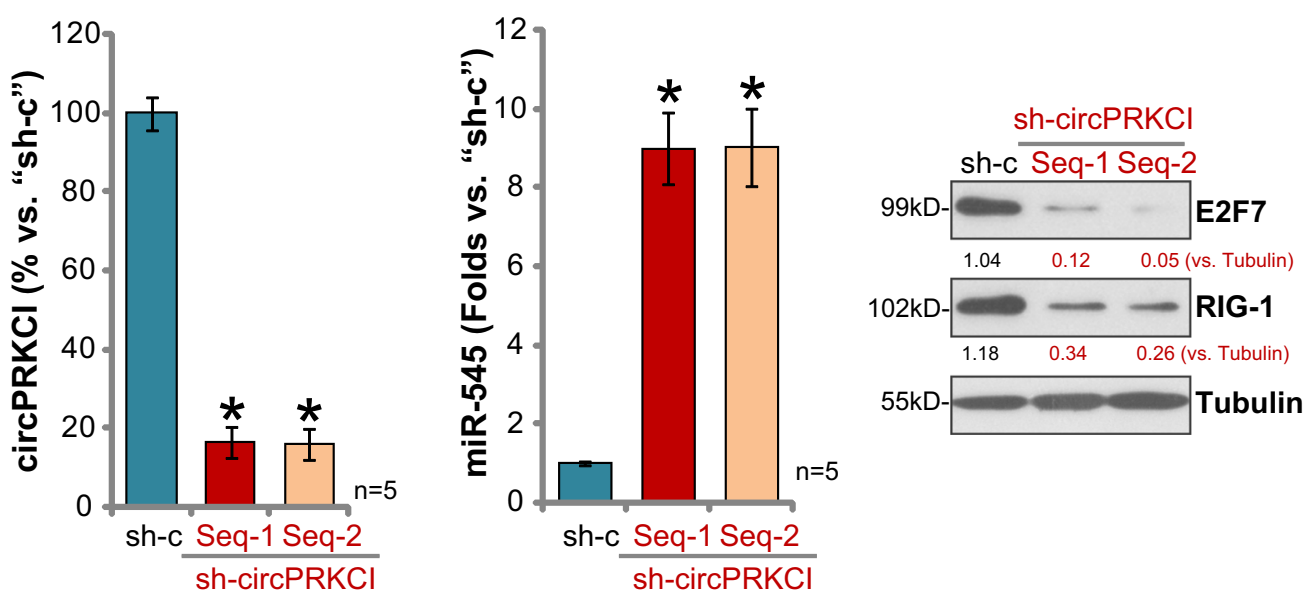

Fig. 6 circPRKCI shRNA inhibits orthotopic A172 glioma growth in mice. Same number of A172 glioma cells, expressing circPRKCl shRNA ("Seq$1 /$ Seq-2") or scramble non-sense control shRNA ("sh-c"), were intracranially injected into the brain of SCID mice (5-6 week old, eight mice per group), after 21 days the animals were sacrificed and tumor volumes (a), tumor weights $(\mathbf{b})$, and mouse body weights (c) recorded. Tumor tissue lysates were analyzed by qPCR and western blotting assays to test listed genes (d-f). E2F7 and RIG-1 protein expression was quantified (f). Error bars stand for mean \pm standard deviation $(\mathrm{SD}, n=5)$. ${ }^{*} P<0.05$ vs. "sh-c" tumors

of subcutaneous and orthotopic A172 gliomas was significantly inhibited by circPRKCI silencing. These results suggest that circPRKCI could be an important and novel therapeutic target of glioma.

Recent studies have shown that miR-545 is a tumorsuppressive miR in human cancers ${ }^{16,28,29}$. Du et al., have shown that miR-545 can inhibit human lung cancer cells $^{29}$. In human pancreatic ductal adenocarcinoma cells, miR-545 inhibits RIG-1 expression and cancer cell growth $^{28}$. In lung cancer cells circPRKCI promotes cancer cell progression by sponging miR-545 ${ }^{16}$. In this study, we show that miR-545 inhibition or knockout promoted A172 cell progression. Conversely, forced overexpression miR-545 inhibited A172 cell survival and proliferation.
Therefore, miR-545 plays a tumor-suppressive role in human glioma cells.

Two primary miR-545 targets are RIG-1 and E2F7 in cancer cells ${ }^{16,26,28,34}$. RIG-I is an intracellular viral RNA sensor, whose activation could initiate host innate immune response to increase type I IFN production ${ }^{35}$. Recent studies have proposed a role of RIG-1 in cancer progression. For instance, Song et al., demonstrated that high RIG-I protein level in pancreatic ductal adenocarcinoma tissues is correlated with shorter survival ${ }^{28}$. Two cancer studies have discovered that miR-545 inhibits human cancer progression by targeting RIG- ${ }^{28,34}$. E2F7 is a relatively novel transcription factor, regulating cell cycle by inhibiting expression of G1-S genes ${ }^{36,37}$. E2F7 could 
form a heterodimer with E2F1 and recruits the corepressor C-terminal-binding protein $(\mathrm{CtBP})^{16}$. Recent studies have implied that downregulation of E2F7 could induce cancer cell apoptosis ${ }^{38,39}$. Importantly, E2F7 could be an independent prognostic factor of gliomas, whose overexpression predicts poor prognosis in glioma patients ${ }^{40}$. Thus, E2F7 could be a novel therapeutic target of human glioma ${ }^{40}$. E2F7 is one key mRNA target of miR$545^{16}$. Here, in A172 and primary human glioma cells ectopic miR-545 overexpression significantly downregulated RIG-1 and E2F7, both were upregulated with miR-545 inhibition.

circPRKCI could sponge miR-545 and possible other tumor-suppressive miRNAs ${ }^{16}$. The results of the current study indicate that miR-545 is the primary target of circPRKCI in glioma cells. RIP assay results show that circPRKCI and miR-545 were both efficiently pulled down by anti-Ago2 antibody in A172 glioma cells. miR-545 levels were significantly increased in circPRKCI-silenced A172 cells, with its targets, E2F7 and RIG-1, downregulated. Importantly, exogenous overexpression of miR545 by a lentiviral construct potently inhibited A172 cell progression, mimicking circPRKCI shRNA-induced activity. Conversely, miR-545 inhibition, via LV-antagomiR-545, abolished circPRKCI silencing-induced antiA172 cell activity. Significantly, miR-545 inhibition or knockout (by CRISPRC/Cas9 method) promoted A172 cell progression. Remarkably, neither circPRKCI shRNA nor circPRKCI overexpression was effective in the miR545-KO A172 cells. In the circPRKCI-silenced subcutaneous and orthotopic A172 xenograft tumor tissues, miR-545 levels were significantly upregulated, correlating with downregulation of its targets, RIG-1 and E2F7. Finally, we show that in human glioma tissues and cells, circPRKCI upregulation correlates with miR-545 downregulation. These results indicate that circPRKCI promotes glioma cell progression possibly by sponging miR545. miR-545 should be the direct target of circPRKCI in glioma cells.

\section{Conclusion}

circPRKCI promotes human glioma cell progression possibly by inhibiting miR-545. Targeting circPRKCImiR-545 cascade could be a novel strategy to inhibit human glioma.

\section{Acknowledgements}

This work was supported by the Medicine and Health Grant from Wenzhou Bureau of Science and Technology (Y20180213). The funders had no role in study design, data collection and analysis, decision to publish, or preparation of the manuscript.

\section{Author details}

${ }^{1}$ Department of Chemoradiation Oncology, The first affiliated hospital of Wenzhou Medical University, Wenzhou, Zhejiang, P.R. China. ${ }^{2}$ Department of
Radiology, The First Affiliated Hospital of Wenzhou Medical University, Wenzhou, Zhejiang, P.R. China

\section{Author contributions}

All listed authors designed the study, performed the experiments and the statistical analysis, and wrote the manuscript. All authors have read the manuscript and approved the final version.

Conflict of interest

The authors declare that they have no conflict of interest.

\section{Publisher's note}

Springer Nature remains neutral with regard to jurisdictional claims in published maps and institutional affiliations.

Received: 11 May 2019 Revised: 26 July 2019 Accepted: 29 July 2019 Published online: 13 August 2019

\section{References}

1. Westphal, M. \& Lamszus, K. The neurobiology of gliomas: from cell biology to the development of therapeutic approaches. Nat. Rev. Neurosci. 12, 495-508 (2011).

2. Siegel, R., Naishadham, D. \& Jemal, A. Cancer statistics, 2012. CA Cancer J. Clin. 62, 10-29 (2012).

3. Siegel, R., Ma, J., Zou, Z. \& Jemal, A. Cancer statistics, 2014. CA Cancer J. Clin. 64 9-29 (2014)

4. Khasraw, M. \& Lassman, A. B. Neuro-oncology: late neurocognitive decline after radiotherapy for low-grade glioma. Nat. Rev. Neurol. 5, 646-647 (2009).

5. Pollack, I. F. Neuro-oncology: therapeutic benefits of reirradiation for recurrent brain tumors. Nat. Rev. Neurol. 6, 533-535 (2010).

6. Wang, Y. \& Jiang, T. Understanding high grade glioma: molecular mechanism, therapy and comprehensive management. Cancer Lett. 331, 139-146 (2013).

7. Neal, J. W., Gainor, J. F. \& Shaw, A. T. Developing biomarker-specific end points in lung cancer clinical trials. Nat. Rev. Clin. Oncol. 12, 135-146 (2015).

8. Rosell, R. \& Karachaliou, N. Lung cancer in 2014: optimizing lung cancer treatment approaches. Nat. Rev. Clin. Oncol. 12, 75-76 (2015).

9. Keith, R. L. \& Miller, Y. E. Lung cancer chemoprevention: current status and future prospects. Nat. Rev. Clin. Oncol. 10, 334-343 (2013).

10. Chen, L., Dzakah, E. E. \& Shan, G. Targetable long non-coding RNAs in cancer treatments. Cancer Lett. 418, 119-124 (2018).

11. Hou, L. D. \& Zhang, J. Circular RNAs: an emerging type of RNA in cancer. Int J. Immunopathol. Pharmacol. 30, 1-6 (2017)

12. Nie, J. H., Li, T. X., Zhang, X. Q. \& Liu, J. Roles of non-coding RNAs in norma human brain development, brain tumor, and neuropsychiatric disorders. Noncoding RNA 5, 36 (2019).

13. Li, Y. et al. Circular RNA as a biomarker for cancer: a systematic meta-analysis. Oncol. Lett. 16, 4078-4084 (2018).

14. Zhao, Z. J. \& Shen, J. Circular RNA participates in the carcinogenesis and the malignant behavior of cancer. RNA Biol. 14, 514-521 (2017).

15. Meng, $X$. et al. Circular RNA: an emerging key player in RNA world. Brief Bioinform. 18, 547-557 (2017).

16. Qiu, M. et al. The circular RNA circPRKCl promotes tumor growth in lung adenocarcinoma. Cancer Res. 78, 2839-2851 (2018).

17. Shi, N. et al. Circular RNA circ-PRKCl functions as a competitive endogenous RNA to regulate AKT3 expression by sponging miR-3680-3p in esophageal squamous cell carcinoma. J. Cell Biochem. 120, 10021-10030 (2019).

18. Qi, S. X. et al. Role and mechanism of circ-PRKCl in hepatocellular carcinoma. World J. Gastroenterol. 25, 1964-1974 (2019).

19. Liu, Y. Y. et al. microRNA-200a downregulation in human glioma leads to Galphai1 over-expression, Akt activation, and cell proliferation. Oncogene 37, 2890-2902 (2018).

20. Jing, D., Yinzhu, L., Jinjing, P., Lishuang, L. \& Guozhuan, Z. Targeting ninjurin 2 by miR-764 regulates hydrogen peroxide ( $\mathrm{H} 2 \mathrm{O} 2)$-induced neuronal cell death Biochem Biophys. Res Commun. 505, 1180-1188 (2018).

21. LeBlanc, A. C., Xue, R. \& Gambetti, P. Amyloid precursor protein metabolism in primary cell cultures of neurons, astrocytes, and microglia. J. Neurochem. $\mathbf{6 6}$ 2300-2310 (1996) 
22. Kaushal, V. et al. Neuronal NLRP1 inflammasome activation of Caspase-1 coordinately regulates inflammatory interleukin-1-beta production and axonal degeneration-associated Caspase-6 activation. Cell Death Differ. 22, 1676-1686 (2015).

23. Shao, N. Y. et al. MicroRNA-29a-3p downregulation causes Gab1 upregulation to promote glioma cell proliferation. Cell Physiol. Biochem. 48, 450-460 (2018).

24. Yu, H., Chen, Y. \& Jiang, P. Circular RNA HIPK3 exerts oncogenic properties through suppression of miR-124 in lung cancer. Biochem. Biophys. Res. Commun. 506, 455-462 (2018).

25. Zhang, B., Lu, H. Y., Xia, Y. H., Jiang, A. G. \& Lv, Y. X. Long non-coding RNA EPIC1 promotes human lung cancer cell growth. Biochem. Biophys. Res. Commun. 503, 1342-1348 (2018).

26. Cheng, Q., Cao, X., Xue, L., Xia, L. \& Xu, Y. CircPRKCI-miR-545/589-E2F7 axis dysregulation mediates hydrogen peroxide-induced neuronal cell injury. Biochem. Biophys. Res Commun. 514, 428-435 (2019).

27. Agnihotri, S. et al. Alkylpurine-DNA-N-glycosylase confers resistance to temozolomide in xenograft models of glioblastoma multiforme and is associated with poor survival in patients. J. Clin. Invest. 122, 253-266 (2012).

28. Song, B. et al. miR-545 inhibited pancreatic ductal adenocarcinoma growth by targeting RIG-I. FEBS Lett. 588, 4375-4381 (2014).

29. Du, B. et al. MicroRNA-545 suppresses cell proliferation by targeting cyclin D1 and CDK4 in lung cancer cells. PLoS One 9, e88022 (2014).

30. Yu, C. Y. et al. The circular RNA circBIRC6 participates in the molecular circuitry controlling human pluripotency. Nat. Commun. 8, 1149 (2017).

31. Wang, $K$. et al. Circular RNA mediates cardiomyocyte death via miRNAdependent upregulation of MTP18 expression. Cell Death Differ. 24, 1111-1120 (2017).
32. Zheng, Q. et al. Circular RNA profiling reveals an abundant circHIPK3 that regulates cell growth by sponging multiple miRNAs. Nat. Commun. 7, 11215 (2016).

33. Ma, Y., Zhang, X., Wang, Y. Z., Tian, H. \& Xu, S. Research progress of circular RNAs in lung cancer. Cancer Biol. Ther. 20, 123-129 (2019).

34. Liu, Z. et al. Ftx non coding RNA-derived miR-545 promotes cell proliferation by targeting RIG-I in hepatocellular carcinoma. Oncotarget 7, 25350-25365 (2016).

35. Yoneyama, M. \& Fujita, T. Function of RIG-H-like receptors in antiviral innate immunity. J. Biol. Chem. 282, 15315-15318 (2007).

36. Liu, B., Shats, I., Angus, S. P., Gatza, M. L. \& Nevins, J. R. Interaction of E2F7 transcription factor with E2F1 and C-terminal-binding protein (CtBP) provides a mechanism for E2F7-dependent transcription repression. J. Biol. Chem. 288 24581-24589 (2013)

37. Di Stefano, L., Jensen, M. R. \& Helin, K. E2F7, a novel E2F featuring DPindependent repression of a subset of E2F-regulated genes. EMBO J. 22, 6289-6298 (2003)

38. Liang, R. et al. SNHG6 functions as a competing endogenous RNA to regulate E2F7 expression by sponging miR-26a-5p in lung adenocarcinoma. Biomed. Pharmacother. 107, 1434-1446 (2018).

39. Wang, C., Li, S., Xu, J., Niu, W. \& Li, S. microRNA-935 is reduced in non-smal cell lung cancer tissue, is linked to poor outcome, and acts on signal transduction mediator E2F7 and the AKT pathway. Br. J. Biomed. Sci. 76, 17-23 (2019)

40. Yin, W. et al. Elevated E2F7 expression predicts poor prognosis in human patients with gliomas. J. Clin. Neurosci. 33, 187-193 (2016). 